\title{
EXTraS discovery of an X-ray superflare from an L dwarf
}

\author{
Andrea De Luca ${ }^{1,2}$, Beate Stelzer ${ }^{3,4}$, Adam J. Burgasser ${ }^{5}$, Daniele Pizzocaro ${ }^{1}$, Piero Ranalli ${ }^{6}$, Stefanie Raetz $^{3}$, \\ Martino Marelli ${ }^{1}$, Giovanni Novara ${ }^{7}$, Cristian Vignali ${ }^{8,9}$, Andrea Belfiore ${ }^{1}$, Paolo Esposito ${ }^{7}$, Paolo Franzetti ${ }^{1}$, \\ Marco Fumana ${ }^{1}$, Roberto Gilli ${ }^{9}$, Ruben Salvaterra ${ }^{1}$, and Andrea Tiengo ${ }^{7,2}$ \\ ${ }^{1}$ INAF - Istituto di Astrofisica Spaziale e Fisica Cosmica Milano, Via A. Corti 12, 20133 Milano, Italy \\ e-mail: andrea.deluca@inaf.it \\ 2 INFN, Sezione di Pavia, Via A. Bassi 6, 27100 Pavia, Italy \\ 3 Institut für Astronomie and Astrophysik Tübingen, Eberhard-Karls Universität Tübingen, Sand 1 72076, Germany \\ ${ }^{4}$ INAF - Osservatorio Astronomico di Palermo, Piazza del Parlamento 1, 90134 Palermo, Italy \\ 5 Center for Astrophysics and Space Science, University of California San Diego, 9500 Gilman Drive, La Jolla, CA 92092, USA \\ 6 Combient Mix AB, Kyrkogatan 22, 41115 Gothenburg, Sweden \\ 7 Scuola Universitaria Superiore IUSS Pavia, Piazza della Vittoria 15, 27100 Pavia, Italy \\ 8 Dipartimento di Fisica e Astronomia, Università degli Studi di Bologna, Via P. Gobetti 93/2, 40129 Bologna, Italy \\ 9 INAF - Osservatorio di Astrofisica e Scienza dello Spazio di Bologna, Via P. Gobetti 93/3, 40129 Bologna, Italy
}

Received 21 November 2019 / Accepted 7 January 2020

\begin{abstract}
We present the first detection of an X-ray flare from an ultracool dwarf of spectral class L. The event was identified in the EXTraS database of XMM-Newton variable sources, and its optical counterpart, J0331-27, was found through a cross-match with the Dark Energy Survey Year 3 release. Next to an earlier four-photon detection of Kelu-1, J0331-27 is only the second L dwarf detected in $\mathrm{X}$-rays, and much more distant than other ultracool dwarfs with X-ray detections (photometric distance of $240 \mathrm{pc}$ ). From an optical spectrum with the VIMOS instrument at the VLT, we determine the spectral type of J0331-27 to be L1. The X-ray flare has an energy of $E_{\mathrm{X}, \mathrm{F}} \sim 2 \times 10^{33} \mathrm{erg}$, placing it in the regime of superflares. No quiescent emission is detected, and from $2.5 \mathrm{Ms}$ of $X M M$ Newton data we derive an upper limit of $L_{\mathrm{X}, \text { qui }}<10^{27} \mathrm{erg} \mathrm{s}^{-1}$. The flare peak luminosity $\left(L_{\mathrm{X}, \text { peak }}=6.3 \times 10^{29} \mathrm{erg} \mathrm{s}^{-1}\right)$, flare duration $\left(\tau_{\text {decay }} \approx 2400 \mathrm{~s}\right)$, and plasma temperature $(\approx 16 \mathrm{MK})$ are similar to values observed in X-ray flares of $\mathrm{M}$ dwarfs. This shows that strong magnetic reconnection events and the ensuing plasma heating are still present even in objects with photospheres as cool as $\sim 2100 \mathrm{~K}$. However, the absence of any other flares above the detection threshold of $E_{\mathrm{X}, \mathrm{F}} \sim 2.5 \times 10^{32} \mathrm{erg}$ in a total of $\sim 2.5 \mathrm{Ms}$ of X-ray data yields a flare energy number distribution inconsistent with the canonical power law $\mathrm{d} N / \mathrm{d} E \sim E^{-2}$, suggesting that magnetic energy release in J0331-27 - and possibly in all L dwarfs - takes place predominantly in the form of giant flares.
\end{abstract}

Key words. X-rays: stars - stars: late-type - stars: activity - stars: flare - stars: coronae

\section{Introduction}

Time-domain astronomy has uncovered a new class of stellar "superflares" with bolometric energies higher than $10^{33} \mathrm{erg}$ (Maehara et al. 2012), events that significantly influence planetary habitability (Armstrong et al. 2016; Lingam \& Loeb 2017) and may have been imprinted on meteoritic chemical abundances in our own solar system (Mishra \& Marhas 2019).

The most surprising superflare discoveries come from the low-mass end of the main sequence, the ultracool dwarfs (UCDs) of spectral type L. Four white-light superflares from L dwarfs have been found in All Sky Automated Survey for Supernovae (Schmidt et al. 2016), Kepler (Gizis et al. 2017; Paudel et al. 2018) and Next Generation Transient Survey data (Jackman et al. 2019), each extremely bright (up to $\Delta V=-11$ ), releasing up to $4 \times 10^{34} \mathrm{erg}$ of energy. These bursts are comparable to the strongest flares in FGKM stars, and are remarkable given that most tracers of quiescent magnetic emission ( $\mathrm{H} \alpha$ and $\mathrm{X}$-ray) decline in the $\mathrm{L}$ dwarf sequence (Schmidt et al. 2015; Stelzer et al. 2006a). This decline is the result of increasingly neutral photospheres (Mohanty et al. 2002) and possibly of changes in magnetic reconnection (Mullan 2010) or dynamo processes (Cook et al. 2014). Furthermore, while young stars are typically the most magnetically active, three of the superflare L dwarfs appear to be older field stars. What enables these dim stars to undergo one of the most dramatic stellar outbursts, and the frequency of such events, remains a mystery.

All of the L dwarf superflares reported to date have been detected in white-light observations. In the standard flare scenario (e.g. Cargill \& Priest 1983) white-light flares are produced in the lower atmosphere following the bombardment with particles that are accelerated in magnetic reconnection events. The ensuing heating of the chromosphere leads to upwards plasma motions and, subsequently, to an X-ray flare from the hot matter confined in coronal magnetic loops. Even for the Sun, observationally associating X-ray and optical flares has remained difficult (Hao et al. 2017). For L dwarfs the lack of simultaneous multi-band observations has so far prevented tests of the solar flare scenario.

Here we report on the first detection of an X-ray superflare emitted by a distant ( $240 \mathrm{pc})$ early L-type dwarf. Previously, only one other L dwarf had been detected in (quiescent) X-rays, 
the binary system Kelu $1 \mathrm{AB}$ at $19 \mathrm{pc}$ (Audard et al. 2007). Our results were obtained within the context of Exploring the X-ray Transient and variable Sky ${ }^{1}$ (EXTraS), an EU-funded project aimed at extracting and characterising all temporal information stored in serendipitous XMM-Newton data (De Luca et al. 2016, De Luca et al., in prep.).

This Letter is organised as follows: in Sect. 2 we describe the discovery of the flare; Sects. 3-5 give temporal and spectral properties of the flare, set constraints on the flaring rate and on the quiescent X-ray emission of the source, and give results on the spectral classification of the dwarf and on its properties. All results are discussed in Sect. 6.

\section{The X-ray flare of J0331-27}

A large catalogue listing 11945 candidate ultracool dwarfs of the $\mathrm{L}$ and $\mathrm{T}$ spectral classes was recently published by Carnero Rosell et al. (2019). This catalogue is based on photometric classification of data from the Dark Energy Survey Year 3 release (Dark Energy Survey Collaboration 2016) covering $\sim 2400$ square degrees down to $i_{\mathrm{AB}}=22$, matched to photometry from the Vista Hemisphere Survey (McMahon et al. 2013) and Wide-field Infrared Survey Explorer (Wright et al. 2010; Mainzer et al. 2011). Of the sources of this catalogue, 515 are located within the field of view of at least one $X M M$ Newton observation. Cross-correlation of the catalogue with the EXTraS database singles out 3XMMJ033158.9-273925 (hereafter J0331-27) as a very interesting case. J0331-27 matches within $11^{\prime \prime} 1$ the position of the L0 candidate Obj. ID $366318917\left(\mathrm{RA}=03^{\mathrm{h}} 31^{\mathrm{m}} 59 \mathrm{~s}\right.$.07, Dec $=-27^{\circ} 39^{\prime} 25^{\prime \prime}$.7; J2000) in Carnero Rosell et al. (2019), and is listed as a variable X-ray source in the EXTraS database. Inspection of light curves in the EXTraS Public Archive ${ }^{2}$ clearly shows an X-ray flare. Moreover, the source is located within the Extended Chandra Deep Field South (ECDFS, Lehmer et al. 2005), one of the most scrutinised portions of the sky, with a large amount of deep multiwavelength data available.

\section{Temporal and spectral properties of the flare}

The X-ray flare from J0331-27 is clearly seen in data collected on 2008 July 5 by all detectors of the European Photon Imaging Camera (EPIC) instrument on board XMM-Newton, namely the pn camera (Strüder et al. 2001) and the two MOS cameras (Turner et al. 2001). The observation (Obs. ID 0555780101) lasted $130 \mathrm{ks}$ and was performed as a part of the XMM-Newton Ultra-deep Survey of the Chandra Deep Field South (XMMCDFS; Comastri et al. 2011; Ranalli et al. 2013). In Fig. 1 we show a background-subtracted light curve combining pn and MOS data, generated using an updated version of the EXTraS software (Marelli et al. 2017). The flare profile is nicely described (reduced $\chi^{2}=0.88,35$ d.o.f.) by a linear rise (rise time of $1600 \pm 300 \mathrm{~s}$ ), peaking at MJD $=54652.8939 \pm 0.0032 \mathrm{UTC}$ (in Barycentric Dynamical Time), followed by an exponential decay of the form $\mathrm{e}^{-t / \tau_{\text {decay }}}$ with $\tau_{\text {decay }}=2400 \pm 400 \mathrm{~s}$. The peak flux is $(9.0 \pm 1.5) \times 10^{-14} \mathrm{erg} \mathrm{cm}^{-2} \mathrm{~s}^{-1}$ and the integrated flare energy flux (fluence), evaluated by integrating the model of the flare time profile, is $\sim 2.7 \times 10^{-10} \mathrm{erg} \mathrm{cm}^{-2}$ (all values refer to the $0.5-2 \mathrm{keV}$ energy range). The best fit model has a continuum level consistent with zero; no significant emission is seen apart from the flare.

\footnotetext{
1 wWW . extras-fp7 .eu

2 https://www88. lamp.le.ac.uk/extras/archive
}

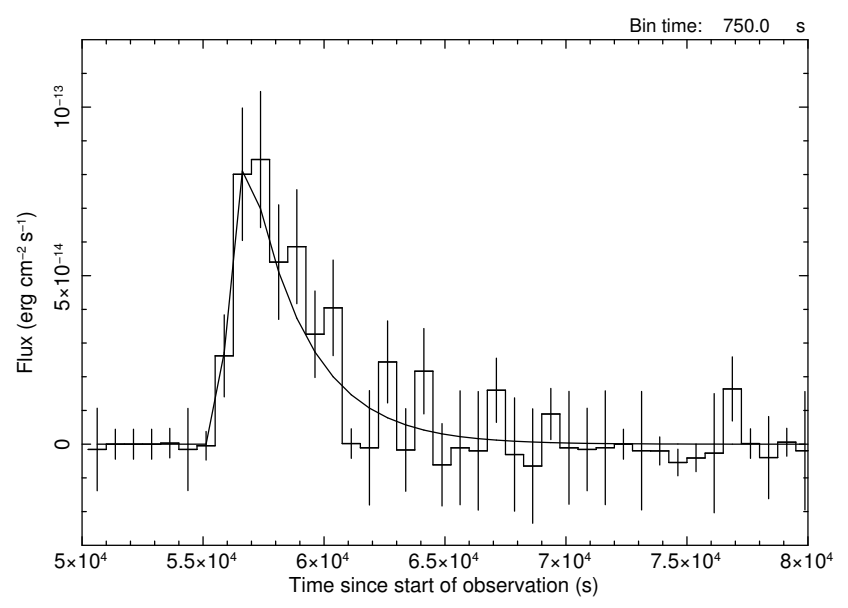

Fig. 1. Background-subtracted light curve of J0331-27 in the $0.2-2 \mathrm{keV}$ energy range combining pn and MOS data. A $30 \mathrm{ks}$ portion of the observation is shown. Count rate has been converted to flux in the $0.5-2 \mathrm{keV}$ energy range using the best fit spectral model (see text). The flare from the source is apparent, and is nicely described by a linear rise and exponential decay model (see text).

To perform spectroscopy of the flare, we extracted source counts from a circle with radius of $15^{\prime \prime}$ and background counts from a nearby, source-free region. We only considered observing times starting $3000 \mathrm{~s}$ before the peak of the flare and extending up to $12000 \mathrm{~s}$ after the peak. Time intervals with high backgrounds were excluded following the prescription by De Luca \& Molendi (2004). The resulting flare spectrum contains $100 \pm 12$ background-subtracted counts from the three EPIC detectors. We generated a response matrix and effective area file using the SAS tasks rmfgen and arfgen. Spectral modelling was performed using the XSPEC v12.10 Software. A good description of the data is given by an optically thin thermal plasma model (apec) with $k T=1.39_{-0.11}^{+0.27} \mathrm{keV}$ and fixed abundance assumed to be 0.3 solar. The absorbing column is consistent with zero $\left(N_{\mathrm{H}}<1.7 \times 10^{20} \mathrm{~cm}^{-2}\right.$ at $\left.1 \sigma\right)$; total Galactic absorption in the direction of the target is indeed very low $\left(N_{\mathrm{H}, \mathrm{Gal}}=6 \times 10^{19} \mathrm{~cm}^{-2}\right.$, HI4PI Collaboration 2016). The goodness of the fit, evaluated as the percentage of Monte Carlo realisations that had Kendall's W-statistic values lower than that of the best fit, is $84 \%$ (based on $10^{4}$ simulations).

The field of J0331-27 was observed 33 times by XMMNewton. We took advantage of this large dataset (total nominal exposure of 3.45 Ms) to search for other flares from J0331-27. No flares were detected within the blind search for transient sources performed by EXTraS, with duration $<10000 \mathrm{~s}$. To set an upper limit to the integrated energy flux of unseen flares, we evaluated the average background level close to the position of the source in individual observations. We followed Ranalli et al. (2013) in their steps for data reduction, event filtering (including soft proton flare screening), and astrometry correction. We excluded observation 0555780101 , in which the flare was detected. Images and exposure maps were generated for each camera and for each observation in the $0.5-2 \mathrm{keV}$ energy range. As a sanity check, we performed aperture photometry by extracting source counts from a circle of $10^{\prime \prime}$ radius and background counts from a contiguous circle with the same radius, combining results from all EPIC cameras for each observation; in no case was significant emission detected from J0331-27. Based on the observed average background rate (assuming a Poissonian regime) and adopting the spectral model best fitting the observed flare of J0331-27, we estimated that an integrated 
energy flux of $\sim 4 \times 10^{-11} \mathrm{erg} \mathrm{cm}^{-2}$ in $5 \mathrm{ks}$ would have produced a $3 \sigma$ excess of counts, which would have triggered the detection algorithm in EXTraS. We note that the 3XMM catalogue lists a detection of J0331-27 in dataset 0108060701. However, the source is detected at the $3 \sigma$ level only in the MOS1 hard band $(4.5-12 \mathrm{keV})$, with upper limits at softer energies not consistent with a thermal spectrum. We disregard this detection as an artefact.

\section{Search for X-ray quiescent emission}

We searched for quiescent emission from J0331-27 by stacking all data from the XMM-CDFS survey, excluding Obs. ID $0555780101(\sim 2.5 \mathrm{Ms}$ good observing time after screening for soft proton flares). Considering only PN data and the $0.5-2 \mathrm{keV}$ band, we extracted 362 counts for J0331-27 from a circular region with a $10^{\prime \prime}$ radius and 349 background counts from an adjacent circular region with the same radius. Assuming a Poisson background distribution, the observed counts can be safely attributed to a background fluctuation.

An upper limit to the quiescent flux of J0331-27 can be calculated by requiring that a detection with the PN camera had at least 50 more counts than in the background region. Formally, such an excess of counts would only have a $0.4 \%$ probability of being due to a background fluctuation. In practice, requiring an excess of 50 counts over the background is consistent with what is observed for other off-axis sources in the XMM-CDFS catalogue (Ranalli et al. 2013). To convert counts to flux, we generated response matrices and effective area files for all XMM-CDFS observations (again excluding Obs. ID 0555780101) and averaged them following Georgantopoulos et al.(2013). Assuming the same spectral model as observed during the flare, we calculate the upper limit to the quiescent emission as $1 \times 10^{-16} \mathrm{erg} \mathrm{s}^{-1} \mathrm{~cm}^{-2}$ in the $0.5-2 \mathrm{keV}$ energy range.

The field of J0331-27 was observed several times by Chandra. It is included in the Extended CDFS, for a total of $\sim 250 \mathrm{ks}$ (Obs. ID 5017 and 5018, PI Brandt). The source was not detected by Lehmer et al. (2005). We retrieved Chandra data and analysed them with the CIAO v4.11 software and CALDB v4.8.3. We reprocessed level 2 data using the chandra_repro script and merged the resulting event files using the reproject_obs script. Using the srcflux script and adopting the spectral shape of the flare emission, we found a $3 \sigma$ upper limit of $5 \times 10^{-16} \mathrm{erg} \mathrm{cm}^{-2} \mathrm{~s}^{-1}$ to the flux of J0331-27 in the $0.5-2 \mathrm{keV}$ energy range. The position of J0331-27 lies also very close to the edge of the field of view in the CDFS 7 Ms survey, at an off-axis angle of $\sim 11$ arcmin, covered with an exposure time (corrected for vignetting) of $\sim 400 \mathrm{ks}$. The source is not detected in these data (Luo et al. 2017). We retrieved the image and exposure map of the survey in the $0.5-2 \mathrm{keV}$ energy range made available by the CDFS team ${ }^{3}$. Following Li \& Ma (1983), we computed the count rate needed to produce a $3 \sigma$ excess in the image at the position of J0331-27 within the PSF area and converted it into flux assuming the observed spectral shape of the flare. The resulting upper limit to the source flux is also $\sim 5 \times 10^{-16} \mathrm{erg} \mathrm{cm}^{-2} \mathrm{~s}^{-1}$.

\section{Classification and properties of the UCD}

\subsection{Spectral classification}

An optical spectrum of J0331-27 was observed with the Visible Multi Object Spectrograph (VIMOS) instrument (Le Fèvre et al.

\footnotetext{
http://personal.psu.edu/wnb3/cdfs/cdfs-chandra.html
}

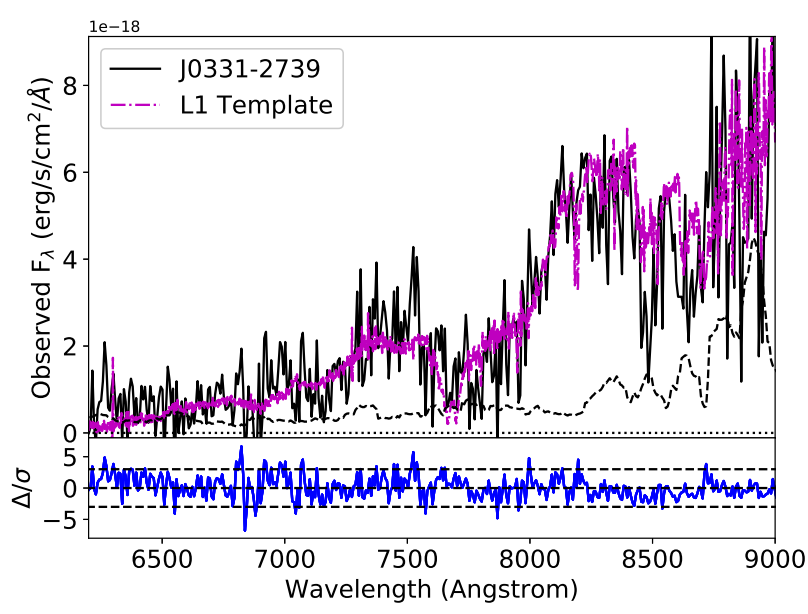

Fig. 2. Top panel: VIMOS spectrum of J0331-27 (black line) compared to an L1 SDSS spectral template from Schmidt et al. (2014, magenta dot-dashed line). The spectral data uncertainty is shown as the dashed line. Bottom panel: difference between the observed data and template $(\Delta)$ normalised to the uncertainty $(\sigma)$.

2003) as part of the VIMOS/VLT Deep Survey of the CDFS (Le Fèvre et al. 2005), where the source is listed with Object ID 38073. Data were reduced using the automatic pipeline developed by the survey team. The spectrum (Fig. 2) shows the characteristic steep rise in continuum across the optical band, with strong absorption features of $\mathrm{K} \mathrm{I}, \mathrm{TiO}, \mathrm{VO}, \mathrm{FeH}, \mathrm{CrH}$ apparent, typical of late-M and L dwarfs (Kirkpatrick et al. 1999). This spectrum was compared to red optical templates of $\mathrm{M}$ and $\mathrm{L}$ dwarfs compiled from Sloan Digital Sky Survey (SDSS) optical spectroscopy by Bochanski et al. (2007) and Schmidt et al. (2014) in the 6500-8000 $\AA$ range using a $\chi^{2}$ statistic. The best fit templates were L1 and (as a close second) L0, with a weighted mean of $\mathrm{L} 0.7 \pm 1.3$, consistent with the photometric classification of Carnero Rosell et al. (2019) ${ }^{4}$. There is no evidence of $\mathrm{H} \alpha$ emission, and low signal-to-noise ratio of the data made it impossible to determine the presence of Li I absorption (a diagnostics of substellarity) or features indicative of low surface gravity (Cruz et al. 2009).

\subsection{Photometric distance}

J0331-27 is not listed in Gaia Data Release 2, thus we have to rely on photometric distance estimates. Carnero Rosell et al. (2019) found $\sim 280 \mathrm{pc}$ from the comparison between the observed near-infrared (NIR) magnitudes and the absolute magnitudes for L0 subtype. Absolute magnitudes are anchored to $M_{W 1}$ and $M_{W 2}$ from Table 14 of Dupuy \& Liu (2012). The other absolute magnitudes are obtained from the colours in Table 3 of Carnero Rosell et al. (2019). Applying the same procedure under the hypothesis of spectral type L1 and averaging over all available bands we obtain a distance of $240_{-20}^{+40} \mathrm{pc}$. This is the value we adopt throughout this paper.

\footnotetext{
The source had been previously classified as an M6 dwarf, or as an unresolved elliptical galaxy at $z \sim 1.9$, by Groenewegen et al. (2002) based on five-band (UBVRI) photometry and morphology (source ID J033159.06-273925.5 in their catalogues). It was also classified as a galaxy by Wolf et al. (2008), based on multi-band photometry (Obj. 51627 in their catalogue).
} 
Table 1. Infrared colours of J0331-27 compared to mean colours of the low-gravity and field-gravity L1 dwarfs from (Faherty et al. 2016).

\begin{tabular}{lccc}
\hline \hline Colour & J0331-27 & $\langle$ Low $-g\rangle$ & $\langle$ Field $\rangle$ \\
\hline$J-H$ & $0.61 \pm 0.15$ & $0.94 \pm 0.06$ & $0.81 \pm 0.14$ \\
$J-K_{s}$ & $1.10 \pm 0.18$ & $1.61 \pm 0.13$ & $1.35 \pm 0.19$ \\
$J-W 1$ & $1.27 \pm 0.19$ & $2.17 \pm 0.20$ & $1.71 \pm 0.21$ \\
$J-W 2$ & $1.80 \pm 0.33$ & $2.55 \pm 0.25$ & $1.97 \pm 0.23$ \\
$H-K_{s}$ & $0.49 \pm 0.19$ & $0.67 \pm 0.11$ & $0.54 \pm 0.13$ \\
$H-W 1$ & $0.66 \pm 0.20$ & $1.21 \pm 0.17$ & $0.91 \pm 0.15$ \\
$H-W 2$ & $1.20 \pm 0.34$ & $1.60 \pm 0.22$ & $1.17 \pm 0.18$ \\
$K_{s}-W 1$ & $0.16 \pm 0.23$ & $0.54 \pm 0.11$ & $0.37 \pm 0.11$ \\
$K_{s}-W 2$ & $0.70 \pm 0.37$ & $0.92 \pm 0.14$ & $0.63 \pm 0.14$ \\
$W 1-W 2$ & $0.54 \pm 0.38$ & $0.38 \pm 0.05$ & $0.26 \pm 0.06$ \\
\hline
\end{tabular}

\subsection{Age characterisation}

The age of J0331-27 is of interest as younger stars tend to be more active, whereas the white light superflare UCDs identified to date appear to be older field objects (Schmidt et al. 2016). Age determinations for individual UCDs are challenging, however, as traditional age metrics such as (quiescent) magnetic activity level and rotation rates appear to decouple from age at the lowest stellar masses and coolest temperatures (e.g. Irwin et al. 2011; Schmidt et al. 2015). Surface gravity-sensitive features in the optical spectra of L dwarfs, such as enhanced VO band and weakened alkali line absorption (Kirkpatrick et al. 2008; Cruz et al. 2009) provide approximate age constraints for sources younger than $\sim 300 \mathrm{Myr}$; unfortunately, the VIMOS spectrum of J0331-27 is too noisy to discern these features. Similarly, kinematics cannot be used as the VIMOS data cannot provide radial velocity information, and no proper motion has been reported for this source.

Colour provides an additional age diagnostic. Faherty et al. (2016) show that NIR colours can be used to distinguish between low-gravity and field-gravity dwarfs, with the latter appearing systematically redder than the mean of the field dwarfs at a given spectral type. Table 1 compares the colours of J0331-27 to the average colours of low surface gravity and field L1 dwarfs reported in Faherty et al. (2016). Rather than being systematically redder, J0331-27 is bluer than field L1 dwarfs, particularly in $J-K_{s}$ and $J-W 1$ colours, consistent with older (high surface gravity) and slightly metal-poor L dwarfs (Zhang et al. 2018). Thus, it is most likely that $\mathrm{J} 0331-27$ is an older field L dwarf.

\section{Discussion}

The association of the flaring source J0331-27 with the L1 dwarf is robust. First, the chance alignment probability is low $\left(<2 \times 10^{-4}\right)$. Second, archival HST images show that the dwarf is the only point-like source within the error circle of the X-ray source. Assuming the two sources to be unrelated would require that the flaring source counterpart have $m_{V}>27$ and $m_{z}>26$. This requires a Galactic flaring X-ray source extremely dim at optical-NIR wavelengths, or an extragalactic transient. Both cases would require peculiar and unlikely scenarios to explain the X-ray emission and temporal behaviour.

The available data do not allow us to precisely constrain the age of J0331-27, although its IR colours indicate that it is likely an older source. Assuming an age $\gtrsim 1 \mathrm{Gyr}$, evolutionary models (Baraffe et al. 2003; Burrows et al. 2001) predict the mass of J0331-27 to be above the hydrogen burning minimum mass limit $\left(M>0.072 M_{\odot}\right)$.
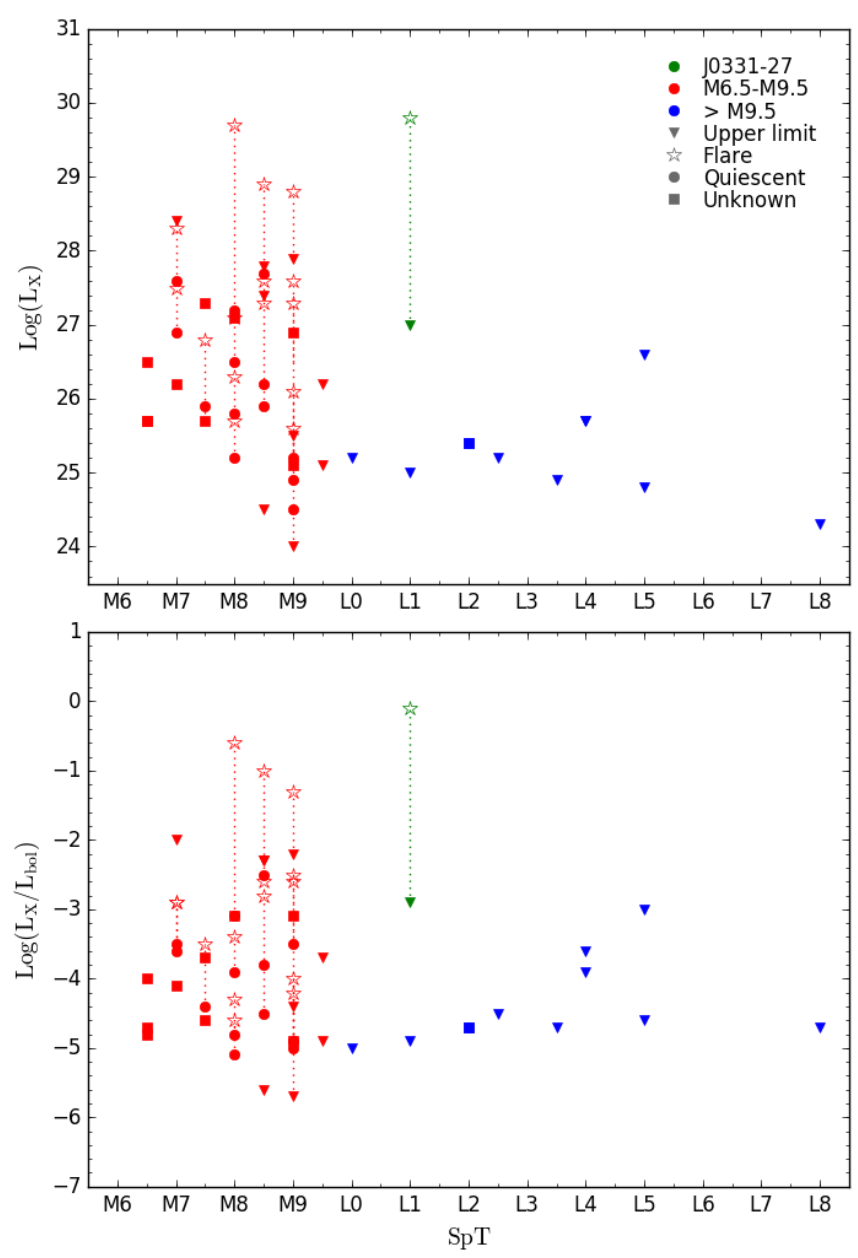

Fig. 3. $L_{\mathrm{X}}$ and $L_{\mathrm{X}} / L_{\text {bol }}$ vs. spectral type for J0331-27 (flare peak luminosity and upper limit on the quiescent luminosity), compared with the other UCDs for which X-ray data are available (from Stelzer et al. 2006a; Williams et al. 2014; Cook et al. 2014 and references therein; Robrade et al. 2010; Gupta et al. 2011).

In Fig. 3 we put the X-ray emission of J0331-27 in context to that of other UCDs. J0331-27 is the first L dwarf to be detected in an X-ray flare. We measure a flare peak luminosity of $\log L_{\mathrm{X} \text {,peak }}=29.8 \mathrm{erg} \mathrm{s}^{-1}$ and $\log \left(L_{\mathrm{X} \text {,peak }} / L_{\text {bol }}\right)=$ -0.1 , similar to X-ray flares observed on late-M dwarfs (e.g. Stelzer et al. 2006b; Hambaryan et al. 2004; Gupta et al. 2011) and actually somewhat larger than the majority of them. The quiescent luminosity is only weakly constrained with an upper limit of $\log L_{\mathrm{X} \text {,qui }}<27.0 \mathrm{erg} \mathrm{s}^{-1}$ and $\log \left(L_{\mathrm{X} \text {,qui }} / L_{\mathrm{bol}}\right)<-2.9$, a result of the large distance of J0331-27 compared with the other UCDs studied in X-rays so far. Similarly, an upper limit on the radio flux of J0331-27 of 7.4 $\mu \mathrm{Jy}$ at $1.4 \mathrm{GHz}$ (Miller et al. 2013), translates into a very weak constraint on the radio luminosity, $\log \mathrm{L}_{v, \mathrm{R}}<14.7 \mathrm{erg} \mathrm{s}^{-1} \mathrm{~Hz}^{-1}$, preventing analysis of the wellestablished deviation of UCDs from the Güdel-Benz radio/X-ray relation (Güdel \& Benz 1993; Berger 2002).

The short observed flare duration (decay timescale $\tau \simeq$ $2400 \mathrm{~s}$ ) is also consistent with those of the X-ray flares on late$M$ dwarfs. This indicates a compact size for the flaring region on the UCD surface (see Stelzer et al. 2006b). Finally, the observed $\mathrm{X}$-ray emitting plasma temperature of $\sim 16 \mathrm{MK}$ is within the range of values reported for late-M dwarf flares. In summary, our observation shows that no qualitative change takes place in the properties of X-ray flares at the bottom of the main sequence down to $T_{\text {eff }} \sim 2100 \mathrm{~K}$. 
The remarkable presence of a single superflare in $\sim 2.5 \mathrm{Ms}$ of XMM-Newton data gives rise to the question on the frequency of such events and of X-ray flares in general on L-type dwarfs. The integrated X-ray flare energy of the event on $\mathrm{J} 0331-27$ is $\log E_{\mathrm{X}, \mathrm{F}}=33.3 \mathrm{erg}$, and the flare frequency is $v\left(\log E_{\mathrm{X}, \mathrm{F}} \gtrsim 33.3\right) \sim 1 / 30 \mathrm{~d}^{-1}$. Based on the sensitivity of the available XMM-Newton observations (assuming a typical flare timescale of $5000 \mathrm{~s}$, see Sect. 3) we estimate that eight flares above $\log E_{\mathrm{X}, \mathrm{F}} \sim 32.4 \mathrm{erg}$ would have been detected if J0331-27 obeyed the canonical power law for the flare energy number distribution, $\frac{\mathrm{d} N}{\mathrm{~d} E_{\mathrm{F}}} \sim E_{\mathrm{F}}^{\alpha}$ with $\alpha \approx-2$ (see references in Argiroffi 2019). The fact that these flares are not seen suggests a nonstandard flare energy distribution for J0331-27.

Contrary to the large flare on the M8 dwarf LP412-32, which was observed simultaneously with the XMM-Newton X-ray instruments and its Optical Monitor (Stelzer et al. 2006b), no contemporaneous optical data is available for the event on J0331-27. However, in view of the similarities of the J0331-27 $\mathrm{X}$-ray flare and the $\mathrm{X}$-ray flares on late-M dwarfs described above, we can use the observed optical-to-X-ray energy ratio of LP412-32 $\left(E_{\mathrm{opt}, \mathrm{F}} \approx E_{\mathrm{X}, \mathrm{F}}\right)$ to estimate an optical counterpart of $E_{\mathrm{opt}, \mathrm{F}} \gtrsim 10^{33} \mathrm{erg}$ for the X-ray superflare on J0331-27. Remarkably, other white-light superflares observed on L dwarfs (without simultaneous X-ray data) show flare energies of the same order (Jackman et al. 2019; Gizis et al. 2017). We note that for a flare on an early $\mathrm{M}$ dwarf observed simultaneously with XMM-Newton and Kepler (KIC 8454353; see Pizzocaro et al. 2019) we also find that the X-ray and optical flare energy are within a factor of two of each other. On the other hand, Guarcello et al. (2019) find significantly higher emission in the optical Kepler band with respect to X-rays for some flares in the Pleiades. Simultaneous optical-X-ray studies of larger samples are required to nail down the relative radiative output of chromosphere and corona, and whether this depends on other stellar parameters.

Systematic searches are also likely to yield better constraints on the frequency of X-ray flares on L dwarfs. Our cross-correlation of the catalogue of UCD candidates by Carnero Rosell et al. (2019) with the XMM-Newton serendipitous source catalogue (3XMM-DR8 ${ }^{5}$, Rosen et al. 2016) yielded two additional close matches between an L dwarf candidate and an X-ray source ${ }^{6}$. Interestingly, both X-ray sources display possible flaring activity, as seen in EPIC light curves produced by the EXTraS software. Optical-NIR spectroscopy is needed to confirm the UCD classification of these objects. Finally, we estimate that the upcoming All-Sky Survey of eROSITA (Merloni et al. 2012) will be able to detect superflares of the size of the event on J0331-27 described here within a volume of $\sim 100 \mathrm{pc}$.

Acknowledgements. This work is based on observations obtained with XMMNewton, an ESA science mission with instruments and contributions directly funded by ESA Member States and NASA. This research has made use of data produced by the EXTraS project, funded by the European Union's Seventh Framework Programme under grant agreement no 607452. We also used observations made by the Chandra X-ray Observatory, and obtained from the Chandra Data Archive. We thank Gian Luca Israel for useful discussions. We acknowledge the computing centre of INAF Osservatorio Astrofisico di Catania for the availability of computing resources and support under the coordination of the CHIPP project. We acknowledge financial support from ASI under ASI/INAF agreement N.2017-14.H.0.

\footnotetext{
5 http://xmmssc.irap.omp.eu/Catalogue/3XMM-DR8/3XMM_ DR8.html

6 3XMM J205205.8-610355, within 1.6" of Obj. ID 185442097 in Carnero Rosell et al. (2019) and 3XMM J232604.0-543340, within $\sim 1.0^{\prime \prime}$ Obj. ID 133082583 in Carnero Rosell et al. (2019).
}

\section{References}

Argiroffi, C. 2019, Astron. Nachr., 340, 284

Armstrong, D. J., Pugh, C. E., Broomhall, A. M., et al. 2016, MNRAS, 455, 3110

Audard, M., Osten, R. A., Brown, A., et al. 2007, A\&A, 471, L63

Baraffe, I., Chabrier, G., Barman, T. S., Allard, F., \& Hauschildt, P. H. 2003, A\&A, 402, 701

Berger, E. 2002, ApJ, 572, 503

Bochanski, J. J., West, A. A., Hawley, S. L., \& Covey, K. R. 2007, AJ, 133, 531

Burrows, A., Hubbard, W. B., Lunine, J. I., \& Liebert, J. 2001, Rev. Mod. Phys., 73, 719

Cargill, P. J., \& Priest, E. R. 1983, ApJ, 266, 383

Carnero Rosell, A., Santiago, B., \& dal Ponte, M. 2019, MNRAS, 2070

Comastri, A., Ranalli, P., Iwasawa, K., et al. 2011, A\&A, 526, L9

Cook, B. A., Williams, P. K. G., \& Berger, E. 2014, ApJ, 785, 10

Cruz, K. L., Kirkpatrick, J. D., \& Burgasser, A. J. 2009, AJ, 137, 3345

Dark Energy Survey Collaboration (Abbott, T., et al.) 2016, MNRAS, 460, 1270

De Luca, A., \& Molendi, S. 2004, A\&A, 419, 837

De Luca, A., Salvaterra, R., Tiengo, A., et al. 2016, in The Universe of Digital

Sky Surveys, eds. N. R. Napolitano, G. Longo, M. Marconi, M. Paolillo, \&

E. Iodice, 42, 291

Dupuy, T. J., \& Liu, M. C. 2012, ApJS, 201, 19

Faherty, J. K., Riedel, A. R., Cruz, K. L., et al. 2016, ApJS, 225, 10

Georgantopoulos, I., Comastri, A., Vignali, C., et al. 2013, A\&A, 555, A43

Gizis, J. E., Paudel, R. R., Mullan, D., et al. 2017, ApJ, 845, 33

Groenewegen, M. A. T., Girardi, L., Hatziminaoglou, E., et al. 2002, A\&A, 392, 741

Guarcello, M. G., Micela, G., Sciortino, S., et al. 2019, A\&A, 622, A210

Güdel, M., \& Benz, A. O. 1993, ApJ, 405, L63

Gupta, A., Galeazzi, M., \& Williams, B. 2011, ApJ, 731, 63

Hambaryan, V., Staude, A., Schwope, A. D., et al. 2004, A\&A, 415, 265

Hao, Q., Yang, K., Cheng, X., et al. 2017, Nat. Commun., 8, 2202

HI4PI Collaboration (Ben Bekhti, N., et al. ) 2016, A\&A, 594, A116

Irwin, J., Berta, Z. K., Burke, C. J., et al. 2011, ApJ, 727, 56

Jackman, J. A. G., Wheatley, P. J., Bayliss, D., et al. 2019, MNRAS, 485, L136

Kirkpatrick, J. D., Reid, I. N., Liebert, J., et al. 1999, ApJ, 519, 802

Kirkpatrick, J. D., Cruz, K. L., Barman, T. S., et al. 2008, ApJ, 689, 1295

Le Fèvre, O., Saisse, M., Mancini, D., et al. 2003, in Society of Photo-Optical Instrumentation Engineers (SPIE) Conference Series, eds. M. Iye, \& A. F. M. Moorwood, Proc. SPIE, 4841, 1670

Le Fèvre, O., Vettolani, G., Garilli, B., et al. 2005, A\&A, 439, 845

Lehmer, B. D., Brandt, W. N., Alexander, D. M., et al. 2005, ApJS, 161, 21

Li, T. P., \& Ma, Y. Q. 1983, ApJ, 272, 317

Lingam, M., \& Loeb, A. 2017, ApJ, 848, 41

Luo, B., Brandt, W. N., Xue, Y. Q., et al. 2017, ApJS, 228, 2

Maehara, H., Shibayama, T., Notsu, S., et al. 2012, Nature, 485, 478

Mainzer, A., Bauer, J., Grav, T., et al. 2011, ApJ, 731, 53

Marelli, M., Tiengo, A., De Luca, A., et al. 2017, ApJ, 851, L27

McMahon, R. G., Banerji, M., Gonzalez, E., et al. 2013, The Messenger, 154, 35

Merloni, A., Predehl, P., Becker, W., et al. 2012, ArXiv e-prints [arXiv:1209.3114]

Miller, N. A., Bonzini, M., Fomalont, E. B., et al. 2013, ApJS, 205, 13

Mishra, R. K., \& Marhas, K. K. 2019, Nat. Astron., 3, 498

Mohanty, S., Basri, G., Shu, F., Allard, F., \& Chabrier, G. 2002, ApJ, 571, 469

Mullan, D. J. 2010, ApJ, 721, 1034

Paudel, R. R., Gizis, J. E., Mullan, D. J., et al. 2018, ApJ, 858, 55

Pizzocaro, D., Stelzer, B., Poretti, E., et al. 2019, A\&A, 628, A41

Ranalli, P., Comastri, A., Vignali, C., et al. 2013, A\&A, 555, A42

Robrade, J., Poppenhaeger, K., \& Schmitt, J. H. M. M. 2010, A\&A, 513, A12

Rosen, S. R., Webb, N. A., Watson, M. G., et al. 2016, A\&A, 590, A1

Schmidt, S. J., West, A. A., Bochanski, J. J., Hawley, S. L., \& Kielty, C. 2014, PASP, 126, 642

Schmidt, S. J., Hawley, S. L., West, A. A., et al. 2015, AJ, 149, 158

Schmidt, S. J., Shappee, B. J., Gagné, J., et al. 2016, ApJ, 828, L22

Stelzer, B., Micela, G., Flaccomio, E., Neuhäuser, R., \& Jayawardhana, R. 2006a, A\&A, 448, 293

Stelzer, B., Schmitt, J. H. M. M., Micela, G., \& Liefke, C. 2006b, A\&A, 460, L35

Strüder, L., Briel, U., Dennerl, K., et al. 2001, A\&A, 365, L18

Turner, M. J. L., Abbey, A., Arnaud, M., et al. 2001, A\&A, 365, L27

Williams, P. K. G., Cook, B. A., \& Berger, E. 2014, ApJ, 785, 9

Wolf, C., Hildebrandt, H., Taylor, E. N., \& Meisenheimer, K. 2008, A\&A, 492, 933

Wright, E. L., Eisenhardt, P. R. M., Mainzer, A. K., et al. 2010, AJ, 140, 1868

Zhang, Z. H., Galvez-Ortiz, M. C., Pinfield, D. J., et al. 2018, MNRAS, 480, 5447 